\title{
On the Mechanism of Gas Transport in Rigid Polymer Membranes
}

\author{
E. R. HENSEMA, ${ }^{*}$ M. H. V. MULDER, ${ }^{\dagger}$ and C. A. SMOLDERS \\ University of Twente, Department of Chemical Technology, P. O. Box 217, 7500 AE Enschede, The Netherlands
}

\begin{abstract}
SYNOPSIS
Conventional polymers are compared as gas separation membrane materials with "tailormade" polymers. The increased permeability of the latter are due to their higher free volume available for gas transport. The increased free volume is associated with the rigidity polymer backbone. Free volume is obtained by subtracting the occupied volume, calculated using group contributions from the polymer specific volume. Wide Angle X-ray techniques are used to obtain average $d$-spacings that are interpreted in terms of average intermolecular space, and that are related to permeability data. These highly permeable rigid polymer membranes have high glass transition temperatures. The physical parameters, that is, $\mathrm{T}_{g}$ and the jump in heat capacity $\left(\Delta \mathrm{C}_{p}\right)$, are obtained with Differential Scanning Calorimetry, and are used to obtain an estimation of free volume. A good correlation for a series of random copoly [ $p, m$-phenylene ( 4 -phenyl) -1,2,4-triazoles] is obtained. A relationship between permeability and a free volume term, which can be estimated from thermodynamic properties, is equally valid for a wide variety of conventional polymers. (c) 1993 John Wiley \& Sons, Inc.
\end{abstract}

\section{INTRODUCTION}

In a previous article, we have reported on the gas transport and separation properties of poly-1,3,4oxadiazoles and poly-1,2,4-triazole membranes. ${ }^{1}$ An increase in permeability appeared to be a function of the diffusivity, only because the latter increased linearly with permeability, whereas solubility remained almost constant.

Permeability and diffusivity could be expressed as a function of the polymer-free volume, using a Doolittle-type equation, previously used by Fujita ${ }^{2}$ and later successfully employed by Lee, ${ }^{3}$ who used this equation to correlate the carbon dioxide and oxygen permeability in various commercial polymers to the polymer specific free volume:

$$
P=S D \exp \left(-B /\left(V_{f}\right)\right)
$$

in which $P$ is the permeability, $S$ is the solubility,

\footnotetext{
† To whom correspondence should be addressed.

* Present address: CIBA-GEIGY AG, Central Research Laboratories, CH-4002 Basel, Switzerland.

Journal of Applied Polymer Science, Vol. 49, 2081-2090 (1993)

(C) 1993 John Wiley \& Sons, Inc. CCC 0021-8995/93/122081-10
}

$B$ is a constant, depending on the penetrant, and $V_{f}$ is the polymer specific free volume. Since $S$ can practically be considered a constant, eq. (1) can be written as:

$$
P=A \exp \left(-B / V_{f}\right)
$$

in which the parameters $A$ and $B$ depend only on the type of gas. Free volume here is defined as

$$
V_{f}=V-V_{0}
$$

where, $V$ is the polymer specific volume and $V_{0}$ is the volume occupied by the polymer chains at $0 \mathrm{~K}$. This volume is assumed to be impermeable for diffusing gas molecules. Lee calculated $V_{0}$ using the relation proposed by Bondi ${ }^{4}$ :

$$
V_{0}=1.3 V_{w}
$$

The Van der Waals volume $\left(V_{w}\right)$ is calculated using a group contribution method. We have used the tabulation of Askadskii ${ }^{5}$ to calculate $V_{w}$. This simple free volume treatment allows a direct insight on the influence of the macromolecular structure on the gas transport properties. The factor 1.3 is, however, arbitrary and may sometimes result in a faulty in- 
terpretation, that is, giving rise to a negative value of $V_{f}^{1}$.

The free volume treatment is gaining interest, since various authors were able to correlate the permeability and diffusivity of various polymer classes to their free volume. For example, Maeda and Paul ${ }^{6}$ used this equation to interpret the reduction in transport in polysulfone and polyphenylene oxide, containing low molecular weight antiplasticisers. The reduction in transport, due to these additives, could be explained in terms of a decrease in free volume. Barbari et al. ${ }^{7}$ related the free volume of polymers based on bisphenol-A to the diffusivity. Muruganandam et al. $^{8}$ and Schmidhauser and Longley ${ }^{9}$ did this for polycarbonates, whereas Min et al. ${ }^{10}$ studied the influence of the tacticity of polymethyl methacrylate using this equation.

Equation (2) implies that for one penetrant, all polymers lie on one straight line. This equation does not, however, account for special polymer/penetrant interactions, differences in macromolecular cohesive forces, and chain flexibilities. Since solubility remains almost constant for one class of related polymers, for example, the poly-1,3,4-oxadiazoles and poly-1,2,4-triazoles studied, it is justifiable to neglect polymer/penetrant interactions in these cases. Vrentas et al. ${ }^{11-13}$ have introduced a modified version of eq. (1), accounting for specific polymer/penetrant interactions. Their approach may better describe the process of permeation in polymers, but their equation contains parameters not directly accessible for the poly-1,3,4-oxadiazoles and poly-1,2,4-triazoles studied.

Free volume is a quantity that depends on the definition used, such as hole-, excess-, configurational-, expansion-, or fluctuation-volume. ${ }^{14}$ The concept of unrelaxed free volume of glassy polymers is, except for gas transport theories, also supplied successfully for understanding and describing many other properties, including impact strength, physical aging, and creep. The aim of this article is to compare the previously reported gas separation properties of poly-1,3,4-oxadiazole and poly-1,2,4-triazole membranes ${ }^{1}$ with other conventional membrane materials and to evaluate other means of free volume determinations.

\section{EXPERIMENTAL}

\section{Materials}

The syntheses, membrane preparation, and gas separation properties of poly-1,3,4-oxadiazoles and poly-1,2,4-triazoles are reported elsewhere. ${ }^{1,15,16}$

\section{Characterization}

\section{Differential Scanning Calorimetry (DSC)}

DSC measurements were performed on a PerkinElmer DSC 4, in combination with a System $4 \mathrm{Mi}$ croprocessor Controller and a Thermal Analysis Data Station (TADS), model 3700. The polymer samples were placed in aluminium sample pans and the temperature was increased at a heating rate of $20^{\circ} \mathrm{C} / \mathrm{min}$, from 100 to $400^{\circ} \mathrm{C}$, under a nitrogen purge gas stream. As glass transition temperature $\left(T_{g}\right)$, the midpoint of the transition was taken, calculated by means of the TADS software. The jump in heat capacity $\left(\Delta C_{p}\right)$ at this point was also calculated using the TADS software.

\section{Wide Angle X-ray Scattering (WAXS)}

Wide angle $\mathrm{X}$-ray diffractograms were recorded on a Philips Röntgen diffractometer, PW1710, using copper radiation $(\mathrm{CuK} \alpha=1.5418 \AA) .^{15}$

\section{RESULTS AND DISCUSSION}

In Figure 1, the permeabilities of conventional polymers, as reported by Lee, ${ }^{3}$ are compared with hexafluoro-substituted aromatic polyimides ${ }^{17,18}$ and the previously reported poly-1,3,4-oxadiazoles and poly1,2,4-triazoles. ${ }^{1}$

According to eq. (1), the carbon dioxide permeability of all polymers should be on one line, but instead, a considerable scattering is found, in which two lines can be drawn: one connecting the commercial polymers studied by Lee, while the other line connects the "tailor-made" polyimides, poly1,3,4-oxadiazoles, and poly-1,2,4-triazoles.

Lee's polymers all have a relatively flexible backbone and a low glass transition temperatures. The polyimides and the poly-1,3,4-oxadiazoles and poly1,2,4-triazoles all have rigid aromatic backbones and higher glass transition temperatures, except for the polyhydrazide ( $\mathrm{PH})$ and the poly (1,4-cyclohexane1,3,4-oxadiazole) ( $\mathrm{CH}$-POD). Figure 1 suggests that rigid polymers exhibit higher permeabilities at the same specific free volumes. In other words, rigid polymers seem to have a higher effective free volume available for the passage of gas molecules. This experimental fact is in contrast with our intuitive feeling that flexible polymers would allow higher permeabilities.

We can again visualize the difference between rigid and flexible polymers using the "spaghetti"model of Dimarzio and Gibbs, ${ }^{19}$ which was previously used to explain the increasing permeability 


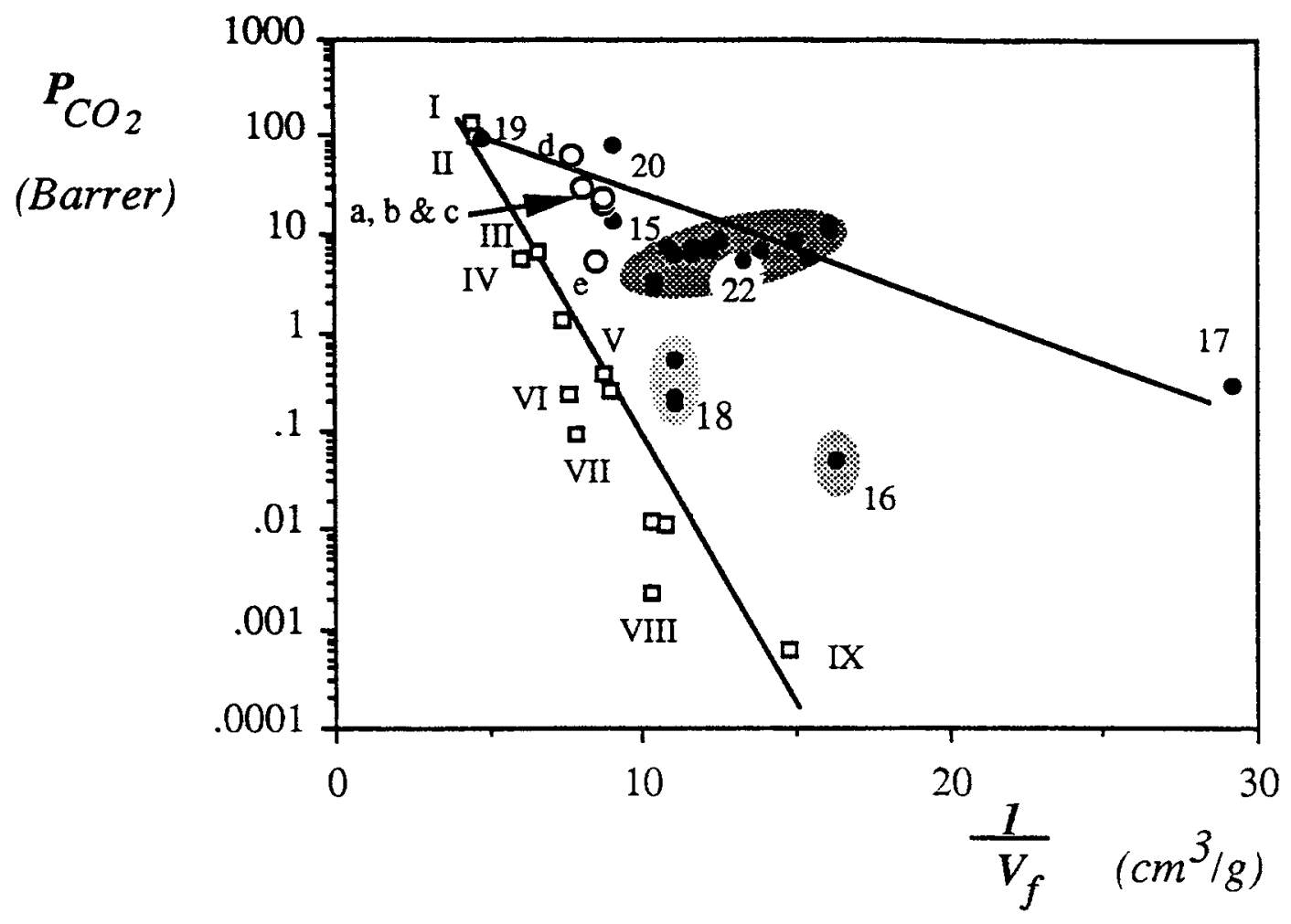

- Conventional polymers studied by Lee (ref. 3) including polybutadiene (I), polyethylene (II), polycarbonate (III), polystyrene (IV), polyethylene terephthalate (V), polymethyl methacrylate (VI), polyvinyl chloride (VII), polymethacrylonitrile (VIII) and polyacrylonitrile (IX).

- Hexafluoro-substituted polyimides.

(a) 6FDA-ODA (ref. 17)

(b) 6FDA-MDA (ref. 17)

(c) 6FDA-IPDA (ref. 17)

(d) $6 F D A-6 F p D A$ (ref. 18)

(e) 6FDA-6FmDA (ref. 18)

- Polyhydrazide, poly-1,3,4-oxadiazoles and poly-1,2,4-triazoles. Numbers and abbreviations are similar to those in ref. $I$

$\begin{aligned} 1-14 & \text { Poly-1,2,4-triazoles including } & 17 & \text { TI-POD } \\ & \text { random copolymers } & 18 & \text { CH-POD } \\ 15 & \text { PT-p/m-Me } & 19 & \text { PIDA-POD } \\ 16 & \text { Polyhydrazide } & 20 & \text { HF-POD } \\ & & 22 & \text { HFIDPE-POD }\end{aligned}$

Figure 1 Carbon dioxide permeability in commercial polymers, hexafluoro-substituted polyimides, poly-1,3,4-oxadiazoles, and poly-1,2,4-triazoles as a function of their reciprocal free volume.

with increasing $p$-phenylene ratio in random poly [ $p$-, $m$-phenylene- (4-phenyl) -1,2,4-triazoles]. ${ }^{1}$ An amorphous matrix of rigid polymer molecules can be compared to a heap of strands of uncooked spaghetti, requiring substantially more free volume than flexible polymers, comparable to cooked 
spaghetti, which can pack into a much tighter form. This difference in packing density will result in a different free volume and in its distribution. From energy and entropy considerations, it follows that a polymer matrix will strive after a narrow free volume distribution during membrane formation. It is easily seen that flexible polymers will be able to achieve a narrow distribution, while rigid polymers will lack this possibility, since these polymers are limited in achieving the same variety of conformations, resulting in a broader free volume distribution, through which the number of larger holes is substantially increased. This is of importance, since it is believed that the larger holes contribute more substantially to the permeation process than smaller ones. This is obvious, since the passage of a permeant with a certain volume requires a minimum hole volume $V_{\min }$.

The existence of free volume distributions has been shown experimentally by means of probe techniques by Victor and Torkelson, ${ }^{20}$ by means of computer Monte Carlo simulations, ${ }^{21}$ and by means of measurements of the jump in specific heat at the glass transition temperature. ${ }^{22}$ Frisch $^{23}$ has discussed the influence of free volume and its distribution on the permeation process.

The hexafluoro substituted polyimides and the poly-1,3,4-oxadiazoles and poly-1,2,4-triazoles are of interest as new membrane materials, since they allow higher permeabilities in combination with higher selectivities. Their increased permeability is a result of their rigidity, resulting in an increased free volume available for gas transport.

Figure 1 shows that the free volume determination, by means of density measurements, and calculation of Van der Waals volumina, by means of group contribution methods, yield an approximate average free volume value. A more accurate and correct determination of the free volume, available for permeation of gas molecules, is highly desirable, so that permeability or diffusivity values of various polymers can be described and compared.

Below, a series of methods and techniques to obtain relevant data will be discussed in detail.

\section{Wide Angle X-ray Scattering (WAXS)}

WAXS experiments are often used to obtain average $d$-spacings of a polymer matrix. The $d$-spacing is assumed to be a measure for the openness of the polymer matrix. A higher average $d$-spacing corresponds to higher average intermolecular distances, allowing easy passage of gas molecules. Differences in the average $d$-spacing can be small, although considerable differences in permeabilities may be found. ${ }^{24}$

The WAXS curves of TI-POD and of the random copolymers poly [ $p$-, $m$-phenylene (4-phenyl) -1,2,4triazoles ], are reported elsewhere, ${ }^{15}$ but the average $d$-spacings of these polymers are represented in Table I.

Comparing the average $d$-spacing of TI-POD with those of the PTs shows that the addition of a pendant phenyl group onto the molecular backbone obviously does result in an increased intermolecular distance, which, in turn, allows a higher permeability, due to the increased free volume.

Interpretation of the poly-1,2,4-triazole WAXS spectra is less straightforward. All poly-1,2,4-triazoles exhibit the same average $d$-spacing, although permeability varies over a factor of four. However, a second "hump" can be found in the spectra at a $2 \Theta$ value of $9.5-10$, or a $d$-spacing of 8.9-9.3 $\AA$. The intensity of this "hump," relative to the main "hump," increases with increasing $p$-phenylene content. This suggests that, besides the average $d$ spacing, its distribution at higher $d$-values may be of importance for the interpretation with respect to permeability data.

\section{Glass Transition and Quenching}

Malhotra and Pethrick ${ }^{25}$ have performed positron annihilation studies on polysulfone and polycarbonate and found a free volume consisting of holes with radii of 1.85 and $2.2 \AA$, respectively. The carbon dioxide permeability for both polymers increases with hole sizes being 4.4 and 6.8 Barrer, respectively. Gol'danskii et al. ${ }^{26}$ also have performed positron annihilation studies in combination with permeability

Table I Carbon Dioxide Permeabilities and Average $d$-Spacings of Random Poly (p-, $m$-phenylene)-1,3,4-Oxadiazole and Poly[p-, $m$-phenylene(4-phenyl)-1,2,4-triazoles $]^{15}$

\begin{tabular}{|c|c|c|c|c|}
\hline Polymer & $\begin{array}{c}T_{g} \\
\left({ }^{\circ} \mathrm{C}\right)\end{array}$ & ${ }_{(\text {Barrer })^{a}}^{\mathrm{P}_{\mathrm{CO}_{2}}}$ & $2 \Theta^{\mathrm{b}}$ & $\begin{array}{c}d \text {-Spacing } \\
(\AA)\end{array}$ \\
\hline $\mathrm{TI}^{-P O D}{ }^{\mathrm{c}}$ & - & 0.3 & 26 & 3.5 \\
\hline PT-0/100 & 242 & 2.7 & 21.5 & 4.2 \\
\hline PT-25/75 & 257 & 3.3 & 21.5 & 4.2 \\
\hline PT-50/50 & 279 & 6.7 & 21.5 & 4.2 \\
\hline PT-75/25 & 319 & 10.1 & 21.5 & 4.2 \\
\hline
\end{tabular}

Barrer $=10^{-10} \mathrm{~cm}^{3}(\mathrm{STP}) \mathrm{cm} / \mathrm{cm}^{2} \mathrm{~s} \mathrm{cmHg}$.

${ }^{b}$ WAXS curves are presented in Ref. 15.

${ }^{\mathrm{c}}$ Gas separation properties were only determined for a $\operatorname{poly}(p$, $m$-phenylene-1,3,4-oxadiazole) containing $50 / 50 \mathrm{wt} \% p$-phenylene and $m$-phenylene groups. No glass transition temperature was detected using DSC-techniques. 
experiments and have found a linear relation between free volume and diffusion coefficients of methane and hydrogen in rubbers. Positron annihilation is an appreciated technique, since it is sensitive to the same structural and molecular dynamic features as the permeating gas molecules. ${ }^{26}$

Gebben et al. ${ }^{28}$ have extensively investigated alternating poly $[p-, m$-phenylene (4-phenyl) -1,2,4triazole ], regarding its gas separation properties, and have found an increase in carbon dioxide permeability after heat treatment at $295^{\circ} \mathrm{C}$ for $1 \mathrm{~h}$. The polymer properties, before and after the heat treatment, are reproduced in Table II. These data can be interpreted in terms of increased free volume for a rapidly cooled or quenched polymer sample, as proposed by Kovacs. ${ }^{27}$ The poly-1,2,4-triazole membrane in Table II was cast from a formic acid solution. During evaporation, the polymer solidifies and a homogeneous film is obtained. This process is comparable with slow cooling of a polymer from the rubbery state into the glassy state. Gebben et al. ${ }^{28}$ heated this polymer membrane during $1 \mathrm{~h}$ at $295^{\circ} \mathrm{C}$, after which it is quenched, followed by permeability and selectivity measurements. An increased permeability and glass transition temperature and a decreased density are found after this heat treatment.

During heat treatment, poly [ $p-, m$-phenylene (4phenyl ) -1,2,4-triazole ] also underwent cold crystallization, complicating a quantitative interpretation of this phenomenon. One would expect a lower permeability for a polymer with increased ordering, but the opposite was found ( see Table II). Quenching obviously dominates over the possible effect of cold crystallization on permeability. In Table I, the glass transition temperature of random poly $[p$-, $m$-phenylene (4-phenyl) -1,2,4-triazoles ] are represented, where it can be seen that permeability increases with increasing glass transition temperature.

Table II Carbon Dioxide Permeability, Selectivity $\left(\alpha_{\left(\mathrm{CO}_{2} / \mathrm{CH}_{4}\right)}\right)$ and Physical Properties of a Homogeneous Poly [p-, $m$-phenylene(4-phenyl)1,2,4-triazole] Membrane ${ }^{28}$

\begin{tabular}{llcc}
\hline Property & & $\begin{array}{c}\text { Before Heat } \\
\text { Treatment }\end{array}$ & $\begin{array}{r}\text { After Heat } \\
\text { Treatment }\end{array}$ \\
\hline$P_{\mathrm{CO}_{2}}$ & $(\text { Barrer })^{\mathrm{a}}$ & 8.9 & 9.2 \\
$\alpha_{\left(\mathrm{CO}_{2} / \mathrm{CH}_{4}\right)}$ & & 62.5 & 60.5 \\
$T_{g}$ & $\left({ }^{\circ} \mathrm{C}\right)$ & 270.4 & 275.6 \\
Density & $\left(\mathrm{g} / \mathrm{cm}^{3}\right)$ & 1.243 & 1.234 \\
\hline
\end{tabular}

a Barrer $=10^{-10} \mathrm{~cm}^{3}(\mathrm{STP}) \mathrm{cm} / \mathrm{cm}^{2} \mathrm{~s} \mathrm{~cm} \mathrm{Hg}$.
Van Krevelen ${ }^{29}$ reports an increase in free volume with increasing glass transition temperature for a wide variety of polymers. A reasonable correlation is observed, although considerable scattering is found. Since the Van der Waals volumina of the isomeric random poly [ $p$-, $m$-phenylene (4-phenyl)1,2,4-triazoles ] remains constant (see Table I), the increase of free volume with increasing glass transition temperature is plausible.

The opposite of quenching, sub- $T_{g}$ annealing, results in a decreased free volume and permeability. Chan and Paul ${ }^{30}$ performed Differential Thermal Analysis (DTA) studies, in combination with carbon dioxide sorption experiments, on sub- $T_{g}$ annealed polycarbonates and also used the approach of Kovacs to interpret their results.

The discussion above suggests that parameters describing processes occurring at the glass transition and the glass transition temperature itself, obtained by means of the appropriate techniques, such as DSC, may provide an appropriate means to estimate the free volume.

\section{Glass Transition and Jump in Specific Heat $\left(\Delta C_{p}\right)$}

Various authors have used the change in the specific volume or specific heat at the glass transition as a measure for the free volume. For example, Vrentas et $a l^{31}$ have used thermal expansion coefficients to calculate changes in the free volume of glassy polymers containing low molecular weight diluents. The fractional free volume can be estimated by means of expansion coefficients and glass transition temperatures, using empirical rules as proposed by Boyer and Spencer ${ }^{32}$ and Simha and Boyer, ${ }^{33}$ respectively:

$$
\begin{gathered}
\alpha_{l} \times T_{g}=K_{1}=0.164 \\
\left(\alpha_{l}-\alpha_{g}\right) T_{g}=\Delta \alpha \times T_{g}=K_{2}=0.113
\end{gathered}
$$

where $\alpha_{l}$ and $\alpha_{g}$ are the expansion coefficients in the liquid or rubbery state and glassy state, respectively, and $\Delta \alpha$ is the change in thermal expansivity at $T_{g}$. The expansion coefficients follow from volumetric determinations.

Differential Scanning Calorimetry (DSC) can be used to obtain the specific heat of a polymer in its rubbery and glassy state. At $T_{g}$, a jump in specific heat is observed: $\Delta C_{p}$. Boyer ${ }^{34}$ has proposed a relationship between $T_{g}$ and $\Delta C_{p}$ that is similar to that of the thermal expansion coefficient:

$$
\Delta C_{p} \times T_{g}=K_{3}=25 \mathrm{cal} / \mathrm{g} .
$$


The expansion coefficient $\alpha$ and heat capacity $C_{p}$ are the derivatives of the thermodynamic entities specific volume $V$ and enthalpy $H$. Since $\Delta C_{p}$ is quickly and easily obtained by standard DSC techniques, this parameter may have the potential of being a valuable polymer characteristic for newly synthesized polymers.

Boyer ${ }^{34}$ has plotted $\Delta C_{p} \times T_{g}$ vs. $T_{g}$ for various commercially available polymers and a considerable scattering can be observed. In our case also, a considerable scattering is observed if $\Delta C_{p} \times T_{g}$ is plotted vs. $T_{g}$ for all poly-1,3,4-oxadiazoles and poly-1,2,4triazoles studied. If we limit ourselves, however, to the homologous series of the random poly $[p-, m$ phenylene (4-phenyl) -1,2,4-triazoles ], a fairly good correlation in accordance with eq. (7) is observed.

In Figure 2, the glass transition temperatures, to-
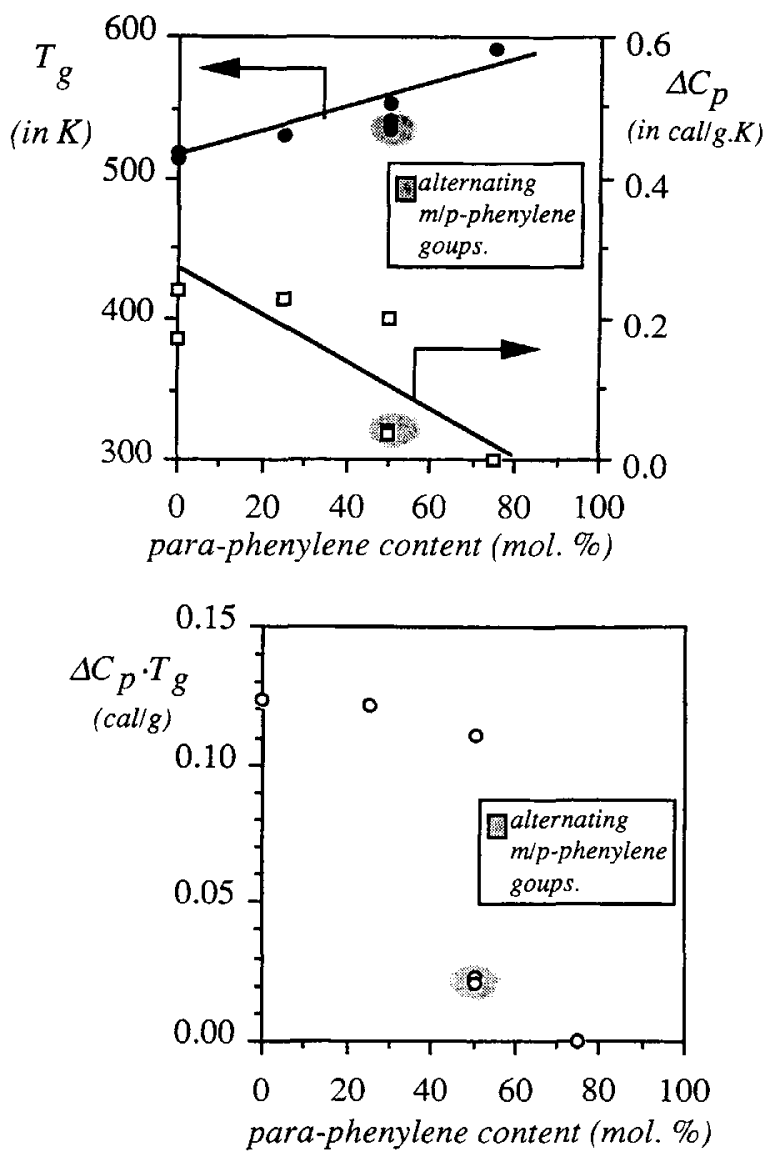

Figure 2 Glass transition temperature and $\Delta C_{p}(a)$ and $\Delta C_{p} \times \mathrm{T}_{g}$ as a function of the $p$-phenylene content in random and alternating poly $[p-, m$-phenylene (4-phenyl) 1,2,4-triazoles]. The glass transition becomes undetectable with DSC techniques at a $p$-phenylene content of $75 \%$. The glass transition was determined with a torsion pendulum in this case and a value of $\Delta C_{p}=0$ was used. ${ }^{15}$ gether with the $\Delta C_{p}$ values (a) and $\Delta C_{p} \times T_{g}$ (b), are plotted as a function of the increasing $p$-phenylene content in random poly $[p-, m$-phenylene (4phenyl ) -1,2,4-triazoles ].

It is shown that the glass transition temperature increases with increasing $p$-phenylene content, whereas $\Delta C_{p}$ decreases with increasing $p$-phenylene content, in accordance with eq. (7). The alternating poly [ $p$-, $m$-phenylene (4-phenyl) -1,2,4-triazole ] show a deviating value for $\Delta C_{p} \times T_{g}$, being only constant for the random poly-1,2,4-triazoles with a $p$ phenylene content less than $75 \%$. The jump in specific heat becomes undetectable at a $p$-phenylene content of $75 \%$, resulting in a $\Delta C_{p} \times T_{g}$ value of zero. The same phenomenon has also been observed for polystyrenes. The $\Delta C_{p}$ value was found to decrease in that case, with an increasing degree of crosslinking. ${ }^{3 \hat{b}}$

A polymer's heat capacity is the result of molecular rotations and vibrations. At the glass transition, the number of rotations and vibrations increases step-wise when the polymer enters the rubbery state since then large chain segments are able to move. In the case of a crosslinked or a stiff macromolecule, as for the poly [ $p$-, $m$-phenylene (4-phenyl) -1,2,4triazole ], with $75 \% p$-phenylene groups, motions in the rubbery state are substantially hindered and the increased rotational and vibrational possibilities are obviously small or negligible since no jump in specific heat is observed.

In a previous article,${ }^{1}$ it was shown that the permeability increased with an increase in glass transition temperature of the homologous series of poly [ $p$-, $m$-phenylene (4-phenyl) -1,2,4-triazoles ]. Since the jump in specific heat decreases with increasing glass transition temperature, permeability or diffusivity can also be expressed as a function of $\Delta C_{p}$.

In Figure 3, the carbon dioxide permeabilities of poly [ $p$-, $m$-phenylene (4-phenyl) -1,2,4-triazoles ] are plotted vs. the jump in heat capacity of the polymers. As a comparison, the carbon dioxide permeability is also plotted vs. $p$-phenylene content in the poly [ $p$-, $m$-phenylene (4-phenyl) -1,2,4-triazoles ]. ${ }^{1}$ A reasonable relation between permeability and $\Delta C_{p}$ is observed, although considerable scattering is observed.

The relationship between $\Delta C_{p}$ and diffusivity was originally observed by $\mathrm{Smit}^{37}$ for a series of hexafluoro-substituted polyimides. The relationship between both quantities was explained in terms of the molecular model of Meares, ${ }^{38}$ originally derived for rubbers. Meares suggested that a diffusional "jump" of a permeant would require a certain amount of 

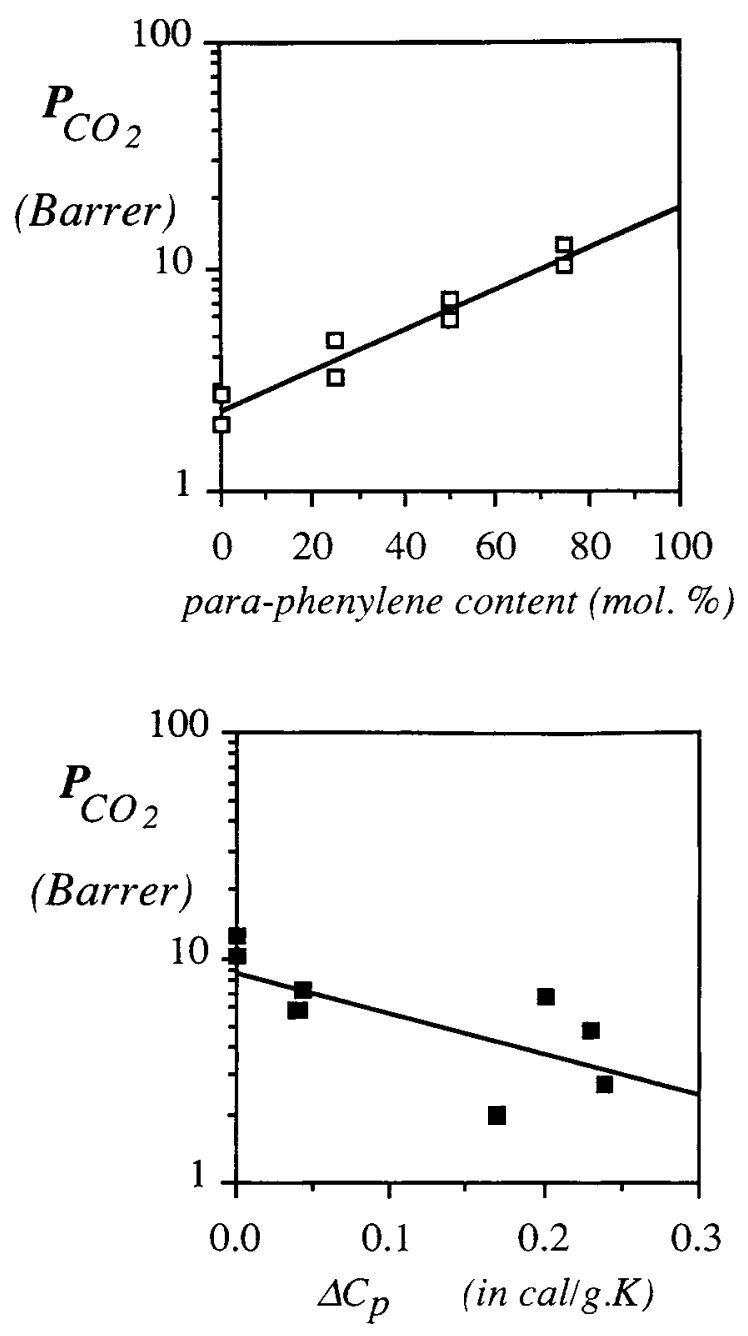

Figure 3 Carbon dioxide permeability as a function of the $p$-phenylene content in poly $[p-, m$-phenylene (4phenyl) -1,2,4-triazoles ] and as a function of the jump in specific heat at the glass transition.

energy, $E_{D}$, and that diffusivity could be described by an Arrhenius-type equation:

$$
D=D_{0} \exp \left(-E_{D} / R T\right)
$$

The magnitude of $E_{D}$ is assumed to depend on the Cohesive Energy Density (CED) of the polymer:

$$
E_{D}=0.25 \pi d^{2} \lambda N_{A V} \mathrm{CED}
$$

Smit calculated the CED of a series of hexafluoro substituted polyimides using the emperical relation between $\Delta C_{p}$ and CED, proposed by Lee ${ }^{39}$ :

$$
\Delta C_{p}=1.25 \mathrm{CED}+2.17(\mathrm{cal} / \mathrm{mol} \mathrm{K})
$$

Meares assumes that, in order to allow the passage of a permeant molecule, contiguous chains or chain segments have "to open up." This treatment suggests that diffusivity is a function of polymer mobility alone and that increasing polymer flexibility will enhance permeability. This is, however, not often the case for glassy polymers. Higher permeabilities are often found for glassy polymers with higher glass transition temperatures, ${ }^{9,17,18}$ as in the case for the poly $[p-, \quad m$-phenylene (4-phenyl)-1,2,4triazoles].

A polymer backbone is considered to be in a frozen state below $T_{g}$ and segmental chain motions are drastically reduced as compared to the rubbery state; gas transport is therefore likely to be not primarily dependent on polymer chain motion.

In addition, a higher glass transition temperature or an increased stiffness was discussed in terms of a larger free volume or a broader free volume distribution. Gas transport in the glassy state is, there-

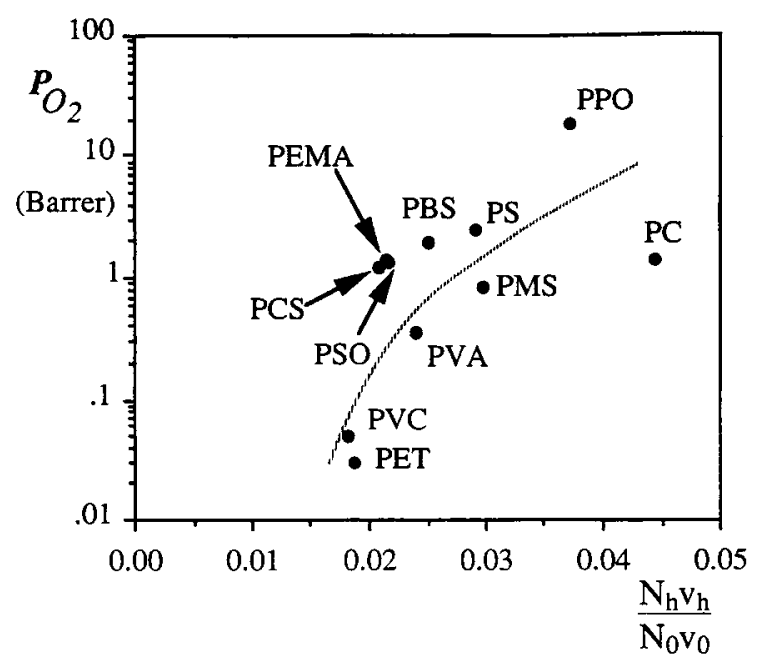

\begin{tabular}{|ll|}
\hline PPO & Poly(phenylene oxide) \\
PS & Polystyrene \\
PMS & Poly( $\alpha$-methylstyrene) \\
PBS & Poly(p-bromostyrene) \\
PCS & Poly(p-chlorostyrene) \\
PMMA & Polymethylmethacrylate \\
PEMA & Poly(ethylmethacrylate) \\
PVA & Polyvinylacetate \\
PVC & Polyvinylchloride \\
PET & Polyethyleenterephthalate \\
PSO & Polysulphone \\
PC & Polycarbonate \\
\hline
\end{tabular}

Figure 4 Oxygen permeability as a function of the ratio nonoccupied/occupied volume $N_{h} v_{h} / N_{0} v_{0}{ }^{38}$ Oxygen permeability data are taken from Ref. 46, for PMS, PCS, and PBS from Ref. 47, and for PPO from Ref. 48. 
fore, assumed to take place via fixed holes. A gas molecule must "find its way" from hole to hole along pathways involving only minor segmental rearrangements. This means that the magnitude of diffusivity or permeability depends largely on the concentration of holes with an appropriate size, able to accommodate a diffusing gas molecule.

Mobility of chain segments in a polymer matrix is, however, strongly related to the polymer free volume. Struik ${ }^{40}$ has visualized this dependence. Free volume has a substantial effect on the mobility in the glassy state and is obviously needed to allow chain segments to move or to rotate "into." For example, the rate of a sub- $T_{g}$ annealing process decreases with decreasing free volume.

This discussion suggests that the amount of free volume is the rate limiting factor for both the transport process and chain mobility. Chen and Edin ${ }^{41}$ came to the same conclusion in their study on gas transport in polycarbonate. A flexible polymer will have an efficiently packed polymer matrix, limiting segmental mobility. A rigid polymer will have a large free volume, allowing increased segmental mobility in the glassy state. Free volume is rate determining both for gas transport and for segmental mobility.

But how can the observed relationship between permeability or diffusivity and the jump in specific heat at the glass transition be explained?

A high $\Delta C_{p}$ means that there is a large difference in heat uptake between the nonequilibrium situation in the glassy state and the equilibrium situation above $T_{g}$ in the rubbery state. This means that $\Delta C_{p}$ may give valuable information concerning the glassy state of the polymer in comparison with the rubbery state. Wunderlich ${ }^{22,35,42}$ has interpreted the physical process behind the jump in heat capacity in terms of the hole theory of Hirai and Eyring. ${ }^{43,44}$ When a glassy polymer is heated, it takes up a certain amount of energy per degree rise in temperature. This amount depends on the heat capacity of the material $C_{p 0}$ :

$$
C_{p, \text { total,glass }}=C_{p 0}
$$

The uptake of energy is used for increased lattice vibrations. In the rubbery, state an extra term, $C_{p h}$ ( $h$ stands for hole), is added to the heat capacity:

$$
C_{p, \text { total, rubber }}=C_{p 0}+C_{p h}
$$

In the rubbery state, molecular rearrangement of neighboring molecules or molecule segments is possible, allowing the creation or disappearance of a hole. The presence of holes is assumed to be necessary for molecular motion.
The second term of the specific heat capacity, $C_{p h}$, is due to the creation of new holes in the rubbery phase. Each hole is characterized by its molar volume, $v_{h}$, and the molar excess energy over the "no hole" situation, $\varepsilon_{h}$.

The part of interest, $C_{p h}$, is due to the change in number of holes with temperature and can be written as:

$$
C_{p h}=\varepsilon_{h}\left(\frac{\partial N_{h}}{\partial T}\right)_{p}
$$

where $N_{h}$ is the number of holes present. Using the equilibrium value of Hirai and Eyring:

$$
\frac{N_{h}}{N_{0}}=\frac{v_{0}}{v_{h}} e^{-\varepsilon_{h} / R T}
$$

where $N_{0}$ is the number chain segments in moles and $v_{0}$ is the volume of one mol of chain segments ( $N_{0} v_{0}=$ occupied volume and $N_{h} v_{h}=$ free volume $)$. Equation (13) becomes:

$$
\Delta C_{p h}=R \frac{v_{0}}{v_{h}}\left(\frac{\varepsilon_{h}}{R T}\right)^{2} e^{-\varepsilon_{h} / R T}
$$

On cooling a polymer from the rubbery into the glassy state, the mechanism responsible for decreasing the number of holes is blocked at the glass transition temperature so that at lower temperatures the number of holes is constant and corresponds to the equilibrium at the transition interval. ${ }^{35}$ At and above $T_{n}$, the additional term $\Delta C_{p h}$ or $\Delta C_{p}$, due to the increase in hole concentration, has to be added. This term should account solely for the rise in heat capacity at $T_{r}$ :

$$
\Delta C_{p}=\Delta C_{p h}=R \frac{v_{0}}{v_{h}}\left(\frac{\varepsilon_{h}}{R T_{g}}\right)^{2} e^{-\epsilon_{h} / R T_{g}}
$$

In eq. (16), $\Delta C_{p}$ is expressed as a complicated function of the molar free volume, the energy for hole formation, and the glass transition temperature. The hole energy, $\varepsilon_{h}$, is a function of the polymer Cohesive Energy Density (CED). Wrasidlo has calculated the hole energies of a wide variety of polymers and found that the hole energy increased linearly with the glass transition temperature. ${ }^{45}$ This is in agreement with our intuitive feeling that, upon increasing rigidity of a polymer system, the possibility of hole formation on passing the glass transition will be restricted. For example, in the case of crosslinked systems or extremely rigid polymers, the difference between the glassy and rubbery state decreases and a sudden in- 
crease in the number of holes at $T_{g}$ is unlikely, due to the large hole energy $\varepsilon_{h}$. Since polymers with high glass transition temperatures often exhibit high permeabilities, this implies that hole formation, in terms of the Hirai and Eyring theory, is not likely or necessary to restrict gas transport through glassy membranes, although it was assumed to be a necessary precondition in the derivation of eq. (9). According to eq. (16), the hole-free volume increases with decreasing $\Delta C_{p}$. This means that permeability or diffusivity will increase with decreasing $\Delta C_{p}$. In the glassy state, the number of holes is fixed and no hole redistributions are likely and it is therefore assumed that gas transport mainly takes place via preexisting holes.

If we neglect the influence of the glass transition temperature on the magnitude of $\Delta C_{p}$, which seems to be allowed since the quotient $\varepsilon_{h} / T_{g}$ is constant, ${ }^{45}$ instead of $\Delta C_{p}, v_{h}^{-1}$ can then be written on the horizontal axis in Figure $3(\mathrm{~b})$, in the case of the poly [ $p-, \quad m$-phenylene (4-phenyl) -1,2,4-triazoles ] studied. This would also mean that eq. (2) can be modified into:

$$
P=A \exp \left(-B \Delta C_{p}\right)
$$

Comparing eqs. (17) and (8) reveals that both the treatment of $\Delta C_{p}$, using the model of Meares and that of Wunderlich, results in a similar type of relation.

Kanig ${ }^{14}$ has also studied the $\Delta C_{p}$ approach extensively and has shown, on the basis of eq. (16), that rigid polymers or polymers with bulky side groups have a higher free volume, being in agreement with the discussion above.

The next question of interest, of course, is that of the general validity of this theory with respect to other classes of polymers. Wrasidlo ${ }^{45}$ has tabulated the related thermodynamic properties, including $\Delta C_{p}, v_{0} / v_{h}$, and $N_{0} / N_{h}$ values, of commercially available polymers. Combined gas transport parameters and thermodynamic properties are only available for a limited number of polymers. Since values for both transport parameters and thermodynamic properties may differ considerably from source to source, we have limited ourselves to three sources. Instead of diffusivity, the oxygen permeability was plotted. Permeability is largely independent of sorption and its increase mainly depends on the increase in diffusivity. ${ }^{1,39}$

Bixler and Sweeting ${ }^{46}$ have published a vast amount of oxygen and carbon dioxide permeabilities of commercially available polymers. Permeabilities were measured at 25 or $30^{\circ} \mathrm{C}$.

In Figure 4, the ratio free volume to occupied vol- ume, $N_{h} v_{h} / N_{0} v_{0}$ [ see eq. (14)] is plotted vs. the oxygen permeability of conventional glassy polymers. Although care should be taken when using data from different sources, a fairly good correlation is observed between the quantities plotted for these glassy polymers, although they belong to a wide variety of different polymer classes. The ratio, $N_{h} v_{h} /$ $N_{0} v_{0}$, is calculated at the glass transition temperature from the thermodynamic quantities $T_{g}, \Delta \alpha$, $\Delta C_{p}$, and CED. ${ }^{45}$ The free volume in the glassy state can be calculated since the free volume at and below $T_{g}$ is constant. ${ }^{45}$ Note that the free volume calculated in this manner is considerably lower, only a few percent, than in the case of the specific free volume and group contributions, as used in Figure 1. The variation in free volume contradicts the assumption of Williams et al. ${ }^{49}$ of a universal value of 0.025 at the glass transition.

Further combined study of gas transport and thermodynamic parameters for a wider range of polymers should reveal the general validity of the relation between permeability or diffusivity and the ratio $N_{h} v_{h} / N_{0} v_{0}$.

\section{CONCLUSIONS}

In this article, conventional polymers were compared with "tailor-made" polymers as gas separation membrane materials. The increased permeability of the latter was shown to be due to their higher free volume that was available for gas transport. The increased free volume is associated with their rigid polymer backbone, not allowing efficient packing, and thereby favoring increased permeability.

Wide Angle X-ray studies show a marked increase in average $d$-spacing between the related aromatic poly-1,3,4-oxadiazole and poly-1,2,4-triazoles, obviously due to the extra phenyl group attached to the polymer backbone of the former. Explaining the increase in permeability with increasing $p$-phenylene content in the poly-1,2,4-triazoles is less straightforward, since all copolymers have the same average $d$-spacing. The "hump" at smaller angles is found to increase with increasing $p$-phenylene content and suggests a difference in free volume distribution at larger intermolecular distances.

The physical processes, occurring at the glass transition and its parameters obtained via DSC techniques, offer a convenient and interesting method to calculate the polymer free volume, using the hole theory of Hirai and Eyring and the derived relationship between the jump in heat capacity and free volume parameters by Wunderlich. A fair relationship was observed for the aromatic copoly- 
1,2,4-triazoles, with increasing $p$-phenylene content between carbon dioxide permeability and jump in heat capacity at the glass transition temperature. Also, for a wide range of well studied conventional polymers, a correlation between free volume and oxygen permeability was found.

Akzo International Research is acknowledged for their financial support.

\section{REFERENCES}

1. E. R. Hensema, J. P. Boom, M. E. R. Sena, M. H. V. Mulder, and C. A. Smolders, Polymer, submitted.

2. H. Fujita, Fortschr. Hochpolym. Forsch., 3, 1-47 (1961).

3. W. M. Lee, Polym. Eng. Sci., 20, 65-69 (1980).

4. A. Bondi, Physical Properties of Molecular Crystals, Liquids, and Glasses, Elsevier, Amsterdam, 1968.

5. A. A. Askadskii, Predictions of Physical Properties of Polymers, in R. A. Pethrick and G. E. Zaikv, Eds., Polymer Yearbook, Vol. 4, Harwood Academic, London, 1987, pp. 93-147.

6. Y. Maeda and D. R. Paul, J. Polym. Sci. Polym. Phys. Ed., 25, 1005-1016 (1987).

7. T. A. Barbari, W. J. Koros, and D. R. Paul, J. Polym. Sci. Polym. Phys. Ed., 26, 709-727 (1988).

8. N. Muruganandam, W. J. Koros, and D. R. Paul, J. Polym. Sci. Polym. Phys. Ed., 25, 1999-2026 (1987).

9. J. C. Schmidhauser and K. C. Longley, J. Appl. Polym. Sci., 39, 2083-2096 (1990).

10. K. E. Min, W. J. Koros, and D. R. Paul, J. Polym. Sci. Polym. Phys. Ed., 26, 1021-1033 (1988).

11. J. S. Vrentas, J. L. Duda, and H.-C. Ling, J. Membrane Sci., 40, 101-107 (1989).

12. J. S. Vrentas, J. L. Duda, and H.-C. Ling, J. Polym. Sci. Polym. Phys. Ed., 23, 275-288 (1985).

13. J. S. Vrentas, J. L. Duda, H.-C. Ling, and A.-C. Hou, J. Polym. Sci. Polym. Phys. Ed., 23, 289-304 (1985).

14. G. Kanig, Kolloid-Z. Z. Polym., 233, 829-845 (1969).

15. E. R. Hensema, J. P. Boom, M. H. V. Mulder, and C. A. Smolders, J. Polym. Sci. Polym. Chem. Ed., submitted.

16. E. R. Hensema, M. E. R. Sena, M. H. V. Mulder, and C. A. Smolders, J. Polym. Sci. Polym. Chem. Ed., submitted.

17. T. H. Kim, W. J. Koros, G. R. Husk, and K. C. O'Brien, J. Membrane Sci., 37, 45-62 (1988).

18. M. R. Coleman and W. J. Koros, J. Membrane Sci., 50, 285-297 (1990).

19. E. A. Dimarzio and J. H. Gibbs, J. Polym. Sci., 40, 121-131 (1959).

20. J. G. Victor and J. M. Torkelson, Macromolecules, 20, 2241-2250 (1987).

21. S. Trohalaki, L. C. DeBolt, and J. E. Mark, Macromolecules, 23, 813-816 (1990).

22. B. Wunderlich, D. M. Bodily, and M. H. Kaplan, J. Appl. Phys., 35, 95-102 (1964).
23. Frisch, J. Polym. Sci. Polym. Lett. Ed., 3, 13-16 (1965).

24. M. Moe, W. J. Koros, H. H. Hoehn, and G. R. Husk, J. Appl. Polym. Sci., 36, 1833-1846 (1988).

25. B. D. Malhotra and R. A. Pethrick, Eur. Pol. J., 19, 457-459 (1983).

26. A. V. Gol'danskii, V. A. Onischchuk, V. P. Shantarovich, V. V. Volkov, and Y. P. Yampol'skii, Khim. Fiz, 7, 616-621 (1988).

27. A. J. Kovacs, J. Polym. Sci., 30, 131-167 (1958).

28. B. Gebben, M. H. V. Mulder, and C. A. Smolders, $J$. Membrane Sci., 46, 29-41 (1989).

29. A. K. Van Krevelen, Properties of Polymers, Third Ed., Elsevier, Amsterdam, 1990, Chap. 4.

30. A. H. Chan and D. R. Paul, Polym. Eng. Sci., 20, 87$94(1980)$.

31. J. S. Vrentas, J. L. Duda, and H.-C. Ling, Macromolecules, 21, 1470-1475 (1988).

32. R. F. Boyer and R. S. Spencer, J. Appl. Phys., 15, 398-405 (1944).

33. R. Simha and R. F. Boyer, J. Chem. Phys., 37, 10031007 (1962).

34. R. F. Boyer, J. Macromol. Sci-Phys., B7, 487-501 (1973).

35. B. Wunderlich, Thermal Analysis, Academic, Boston, 1990, Chap. 4.

36. L. H. Judovits, R. C. Bopp, U. Gaur, and B. Wunderlich, J. Polym. Sci. Polym. Phys. Ed., 24, 27252741 (1986).

37. E. Smit, Ph. D. Thesis, University of Twente, Enschede, The Netherlands, 1991.

38. P. Meares, J. Am. Chem. Soc., 76, 3415-3422 (1954).

39. C. J. Lee, Polym. Eng. Sci., 27, 1015-1017 (1987).

40. L. C. E. Struik, Physical Aging of Amorphous Polymers and Other Materials, Elsevier, Amsterdam, 1978, Chap. 2.

41. S. P. Chen and J. A. Edin, Polym. Eng. Sci., 20, 40$50(1980)$.

42. B. Wunderlich, J. Phys. Chem., 64, 1052-1056 (1960).

43. N. Hirai and H. Eyring, J. Appl. Phys., 29, 810-816 (1958).

44. N. Hirai and H. Eyring, J. Polym. Sci., 37, 51-70 (1959).

45. W. Wrasidlo, Fortschr. Hochpolym. Forsch., 13, 3-99 (1974).

46. H. J. Bixler and O. J. Sweeting, Barrier Properties of Polymer Films, in O. J. Sweeting, Ed., The Science and Technology of Polymer Films, Vol. 2, Wiley, New York, 1971.

47. A. C. Puleo, N. Muruganandam, and D. R. Paul, J. Polym. Sci. Polym. Phys. Ed., 27, 2385-2406 (1989).

48. W. J. Koros, B. J. Story, S. M. Jordan, K. O'Brien, and G. R. Husk, Polym. Eng. Sci., 27, 603-610 (1987).

49. M. L. Williams, R. F. Landel, and J. D. Ferry, J. Am. Chem. Soc., 77, 3701-3707 (1955).

Received October 28, 1991

Accepted January 14, 1993 